Jeffrey M. Statland, MD

Sabrina Sacconi, MD,

$\mathrm{PhD}$

Constantine Farmakidis, MD

Colleen M. Donlin-

Smith, MA

Mina Chung, MD

Rabi Tawil, MD

Correspondence to

Dr. Statland:

Jeffrey_Statland@URMC.

Rochester.edu

\title{
Coats syndrome in facioscapulohumeral dystrophy type 1
}

\author{
Frequency and D4Z4 contraction size
}

[ad

\section{ABSTRACT}

Objective: To investigate the frequency of Coats syndrome and its association with D4Z4 contraction size in patients with facioscapulohumeral muscular dystrophy type 1 (FSHD1).

Methods: We searched a North American FSHD registry and the University of Rochester (UR) FSHD research database, reviewed the literature, and sent surveys to 14 FSHD referral centers in the United States and overseas to identify patients with genetically confirmed FSHD1 with a diagnosis of Coats syndrome.

Results: Out of 357 genetically confirmed patients in a North American FSHD registry and 51 patients in the UR database, 3 patients had a self-reported history of Coats disease $(0.8 \%$; $95 \%$ confidence interval $0.2 \%-2.2 \%)$. In total, we identified 14 patients with FSHD with known genetic contraction size and Coats syndrome confirmed by ophthalmologic examination: 10 from our survey and 4 from the literature. The median age at diagnosis of Coats syndrome was 10 years (interquartile range 14 years). The median D4Z4 fragment size was 13 kilobases (kb) (interquartile range $1 \mathrm{~kb}$ ). One patient was mosaic (55\% $11 \mathrm{~kb}$, and $45 \% 78 \mathrm{~kb})$.

Conclusions: Coats syndrome is a rare extramuscular complication of FSHD1 associated with large D4Z4 contractions. Closer surveillance for retinal complications is warranted in patients with D4Z4 fragments $\leq 15 \mathrm{~kb}$. Neurology ${ }^{\circledR}$ 2013;80:1247-1250

\section{GLOSSARY}

$\mathbf{C l}=$ confidence interval; FSHD1 = facioscapulohumeral muscular dystrophy type $1 ; \mathbf{k b}=$ kilobase; UR $=$ University of Rochester.

Facioscapulohumeral muscular dystrophy type 1 (FSHD1) is caused by loss of a critical number of subtelomeric D4Z4 macrosatellite repeat units on chromosome 4q35. Whereas normal individuals have 11-100 repeats, individuals with FSHD have 1-10 repeats, which translates to D4Z4 EcoRI restriction fragment size between 10 and 38 kilobases $(\mathrm{kb})$ by conventional genetic testing. ${ }^{1}$ Extramuscular manifestations include high-frequency hearing loss and retinal vascular tortuosity, which can progress to a treatable symptomatic condition called Coats syndrome.

The idiopathic form of this condition, Coats disease, is typically unilateral and predominately affects males with an age at onset in the first 2 decades of life. Retinal examination reveals telangiectasias and leakage that can lead to exudative retinal detachment and blindness. ${ }^{2}$ Treatment ranges from laser photocoagulation and cryotherapy in mild disease to vitreoretinal surgery or enucleation in advanced stages. ${ }^{2}$ Coats disease as seen in FSHD (Coats syndrome) can be bilateral, occur at any age, and affect males and females equally.

While more than half of patients with FSHD1 show peripheral retinal vascular abnormalities on fluorescein angiography, the prevalence of Coats syndrome is much lower, and the relationship to the D4Z4 contraction size is unknown. ${ }^{3,4}$ Understanding which patients are most at risk for Coats syndrome is an important clinical question with direct implications for screening and surveillance.

METHODS We performed a cross-sectional database review and a deidentified patient survey of international FSHD1 neuromuscular referral centers. This study was considered exempt after institutional review board review.

From the Departments of Neurology (J.M.S., C.M.D., R.T.) and Ophthalmology (M.C.), University of Rochester Medical Center, Rochester, NY; Centre de Référence Maladies Neuromusculaires (S.S.), Hôpital Archet-CHU de Nice, France; and Department of Neurology (C.F.), Albert Einstein College of Medicine, Bronx, NY.

Go to Neurology.org for full disclosures. Funding information and disclosures deemed relevant by the authors, if any, are provided at the end of the article. 
Registry and database review. We analyzed deidentified data from patients with genetically confirmed FSHD (D4Z4 fragment $<38 \mathrm{~kb}$ ) from the National Registry of FSHD Patients and Family Members $(\mathrm{n}=357)$ and the University of Rochester (UR)-FSHD database $(\mathrm{n}=51)$. Participants were self-selected, from North America, and over 18 years of age (UR-FSHD database), or any age (Registry). We used selfreported history of Coats disease for prevalence estimates. Because data are deidentified, participants may be double represented in the Registry and UR-FSHD database. We compared participants based on D4Z4 deletion size ( $\pm 1 \mathrm{~kb}$ ), sex, and age at diagnosis ( \pm 5 years). The denominator used for point prevalence estimates $(\mathrm{n}=396$ total, $\mathrm{n}=24$ D4Z4 $\leq 15$ $\mathrm{kb}$ ) was the sum of participants from the 2 databases minus possible overlapping subjects $(\mathrm{n}=12)$.

Survey. We sent a one-page deidentified survey to 14 neuromuscular FSHD referral centers in the United States and overseas. In addition, we solicited cases at international meetings, by e-mail, and on a national neuromuscular online forum. Survey respondents reviewed medical records to obtain information about Coats diagnosis and FSHD genetic testing. The Coats stage was modified from the literature and includes the following categories: $1=$ retinal telangiectasia only; $2=$ retinal telangiectasia and exudation; $3=$ exudative retinal detach ment; $4=$ total retinal detachment and glaucoma; and $5=$ advanced end-stage disease. ${ }^{2}$

Literature review. We searched PubMed using the following search terms: facioscapulohumeral muscular dystrophy, FSHD, Coats disease, retinal vascular disease, and retinopathy. Thirty-one articles contained the search terms; 4 articles reported D 4 Z4 contraction size.

Statistical considerations. We performed analysis using SAS version 9.3 (SAS Institute Inc., Cary, NC). Unless otherwise stated, all descriptive analysis is presented as median with quartile data in parenthesis (first quartile $=\mathrm{Q} 1=25 \%$ of data, third quartile $=\mathrm{Q} 3=75 \%$ of data). We presented sex, hearing loss, Coats stage, and sidedness of eye involvement as population frequencies. Prevalence is taken as the number affected divided by the number at risk, with Exact binomial confidence interval (CI).

\section{Table 1 Frequency of Coats syndrome in the FSHD National Registry and UR-FSHD database}

$\begin{array}{lll} & \text { Registry } & \text { UR-FSHD database }{ }^{a} \\ \text { No. } & 357 & 51 \\ \text { Age, } y & 45(33,56) & 50(40,59) \\ \text { M/F } & 175 / 182 & 36 / 15 \\ \text { Age diagnosed with FSHD, y } & 29(17,45) & 32(19,47) \\ \text { D4Z4 allele size, kb } & 25(21,29) & 21(17,29) \\ \text { No. alleles } \leq 15 \text { kb } & 18 & 9 \\ \text { Hearing loss, } n(\%) & 64(17.9) & 12(23.5) \\ \text { Hearing aid, } n(\%) & 25(7.0) & 5(9.8) \\ \text { Coats syndrome, } n \text { (\%) } & 2(0.6)^{b} & 1(2.0)^{\mathrm{c}}\end{array}$

Abbreviations: FSHD = facioscapulohumeral muscular dystrophy; $\mathrm{kb}=$ kilobase; Registry = the National Registry of FSHD Patients and Family Members; UR = University of Rochester. ${ }^{a}$ Age, age diagnosed with FSHD, and D4Z4 allele size are presented as median (Q1 = quartile $1=25 \%$ of data, Q3 = quartile $3=75 \%$ of data).

${ }^{\mathrm{b}}$ The 2 participants are both female: one allele size $26 \mathrm{~kb}$; one first-degree relative allele size $30 \mathrm{~kb}$.

${ }^{\mathrm{c}}$ Survey case 5 in table 2 .
RESULTS Database review. The Registry population $(\mathrm{n}=357$, table 1$)$ was equally divided between the sexes, with a median age at diagnosis of 29 years, and median D4Z4 fragment size of $25 \mathrm{~kb}$, including 18 participants with an allele size $\leq 15 \mathrm{~kb}$. Two participants reported a history of Coats disease (both female, allele sizes $26 \mathrm{~kb}$ and $30 \mathrm{~kb})$. The UR-FSHD database $(\mathrm{n}=51)$ included more men than women $(70.6 \%$ male). The median age at diagnosis was 32 years, with a median D4Z4 fragment size of $21 \mathrm{~kb}$, and 9 participants with allele size $\leq 15 \mathrm{~kb}$. One participant reported a history of Coats disease (female, survey case 5).

The prevalence of Coats syndrome was $0.8 \%$ of the total population ( $95 \%$ CI $0.2 \%-2.2 \%$ ).

Survey and literature review. Ten survey cases and 4 cases in the literature reported $\mathrm{D} 4 \mathrm{Z} 4$ contraction size in patients with FSHD and Coats syndrome confirmed on funduscopy (table 2). Thirteen out of 14 of these cases were female (92.9\%). The median age at diagnosis of FSHD was 12 years, and the median D4Z4 fragment length was $13 \mathrm{~kb}$. One subject was mosaic with $11 \mathrm{~kb}$ (55\%), $78 \mathrm{~kb}$ (45\%, survey case 2). The median age at diagnosis for Coats syndrome was 10 years, but 2 respondents developed symptoms at $\geq 50$ years. Both eyes were affected in $64.3 \%$ of cases and $71.4 \%$ had received therapy. Hearing loss was reported in $57.1 \%$ of cases, with hearing aid use in $35.7 \%$ of cases, median age at first use 2 years.

DISCUSSION Prevalent rates of retinal abnormalities for FSHD1 in the literature varied greatly between studies, from $7 \%$ to $75 \%$; however, only a single patient had visual loss (0.7\%). ${ }^{3-6}$ In addition, a Dutch population survey found 3/256 (1.7\%) patients reporting Coats disease. ${ }^{4}$ This yields a conservative range for symptomatic disease between $0.7 \%$ and $1.7 \%$.

The median allele size in patients with FSHD1 with Coats syndrome was $13 \mathrm{~kb}$, considerably smaller than the median reported in the Registry or UR-FSHD database. Approximately $5 \%-10 \%$ of patients with FSHD type 2 (FSHD2) develop disease by a D4Z4 contractionindependent pathway. Common to both FSHD1 and FSHD2 is relaxation of chromatin structure in the D4Z4 region of chromosome $4 \mathrm{q} 35$ and a specific polymorphism, which leads to release of repression of a putative retrogene, DUX4, believed to be directly toxic to cells. ${ }^{7}$ To our knowledge, no cases of Coats syndrome have been described in patients with FSHD2, although the literature on FSHD2 is limited. Patients with FSHD1 with the largest contractions typically have more severe neuromuscular disease, raising the possibility of a contraction-dependent increase in DUX4 expression. Somatic mosaics tend to have mild neuromuscular manifestations, and so Coats syndrome in a mosaic patient carrying a large D4Z4 contraction (case 2) suggests a differential susceptibility of the retinal vasculature to large 
Table 2 Coats syndrome and D4Z4 fragment size in 14 cases $^{\mathrm{a}}$

\begin{tabular}{|c|c|c|c|c|c|c|c|c|c|c|c|c|c|c|c|}
\hline \multirow[b]{2}{*}{ Case no. } & \multicolumn{10}{|c|}{ Survey } & \multicolumn{4}{|c|}{ Literature } & \multirow[b]{2}{*}{ Total $^{b}(n=14)$} \\
\hline & 1 & 2 & 3 & 4 & 5 & 6 & 7 & 8 & 9 & 10 & $1^{9}$ & $2^{9}$ & $3^{10}$ & $4^{8}$ & \\
\hline Age, y & 14 & 54 & 8 & 51 & 59 & 8 & 23 & 6 & 14 & 52 & 18 & 26 & 5 & 39 & $21(10,48)$ \\
\hline Female & $\mathrm{Y}$ & $\mathrm{Y}$ & $\mathrm{Y}$ & $\mathrm{Y}$ & $\mathrm{Y}$ & $\mathrm{N}$ & $\mathrm{Y}$ & $\mathrm{Y}$ & $\mathrm{Y}$ & $\mathrm{Y}$ & Y & $\mathrm{Y}$ & $\mathrm{Y}$ & $\mathrm{Y}$ & $92.9 \%$ \\
\hline FSHD diagnosis years & 10 & 40 & 2 & 22 & 16 & 5 & 16 & 0.7 & 11 & 12 & 5 & 19 & 1 & 39 & $12(5,18)$ \\
\hline D4Z4 fragment kb & 13 & $11 / 78^{c}$ & 13 & 13 & 10 & 13 & 13 & 13 & 13 & 3 Repeats ${ }^{d}$ & 12 & 12 & 13 & 27 & $13(12,13)$ \\
\hline Coats stage & 3 & 2 & 5 & 2 & 2 & 2 & 2 & 2 & 3 & 2 & 5 & 2 & 5 & 2 & $2(2,3)$ \\
\hline Bilateral & $\mathrm{Y}$ & NS & $\mathrm{N}$ & $\mathrm{Y}$ & $\mathrm{N}$ & $\mathrm{N}$ & NS & $\mathrm{Y}$ & Y & Y & Y & $\mathrm{Y}$ & Y & Y & $64.3 \%$ \\
\hline Therapy for Coats & $\mathrm{Y}$ & $\mathrm{N}$ & $\mathrm{Y}$ & $\mathrm{N}$ & $\mathrm{Y}$ & $\mathrm{Y}$ & $\mathrm{Y}$ & $\mathrm{Y}$ & $\mathrm{Y}$ & $\mathrm{N}$ & Y & $\mathrm{Y}$ & $\mathrm{Y}$ & $\mathrm{N}$ & $71.4 \%$ \\
\hline Hearing loss & $\mathrm{N}$ & $\mathrm{Y}$ & $\mathrm{Y}$ & $\mathrm{Y}$ & $\mathrm{N}$ & $\mathrm{Y}$ & $\mathrm{Y}$ & $\mathrm{N}$ & $\mathrm{N}$ & $\mathrm{N}$ & Y & $\mathrm{Y}$ & $\mathrm{Y}$ & $\mathrm{N}$ & $57.1 \%$ \\
\hline
\end{tabular}

Abbreviations: FSHD = facioscapulohumeral muscular dystrophy; kb = kilobase; NS = not specified.

a Surveys were sent to the following neuromuscular referral centers: University of Rochester Medical Center; Columbia Medical Center; The Kennedy Krieger Institute; University of lowa Hospitals and Clinics; UC Davis Medical Center; The Ohio State University; Children's Hospital of Philadelphia; UT Southwestern; University of Newcastle upon Tyne, UK; Radboud University Nijmegen Medical Centre, the Netherlands; Hôpital Archet-CHU de Nice, France; National Research Council Argentina; University of Sydney, Australia; and St. Michael's Hospital, UK.

${ }^{\mathrm{b}}$ Age, FSHD diagnosis years, D4Z4 fragment kb, age hearing loss, age hearing aid, age Coats, Coats stage: all totals presented are median (Q1 = quartile $1=25 \%$ of data, Q3 = quartile $3=75 \%$ of data).

${ }^{c}$ Mosaic patient allele size $11 \mathrm{~kb}=55 \%, 78 \mathrm{~kb}=45 \%$.

${ }^{\mathrm{d}}$ Allele length not available in kilobases for this patient.

contractions. All cases were $\leq 13 \mathrm{~kb}$, except for literature case $4(27 \mathrm{~kb}){ }^{8}$ This case is atypical with no symptoms or signs of FSHD and retinal changes that were noted incidentally on routine funduscopy. Further testing to exclude a rare false-positive genetic test is warranted given this unusual presentation.?

The predominance of females in the cases presented is striking given the male predominance reported in idiopathic Coats disease, and suggests a unique (genetic, hormonal) susceptibility of the retinal vasculature in females with FSHD1. Although other conditions lead to secondary Coats-like syndromes (retinitis pigmentosa, CTC1 mutation), the pathologic mechanisms and clinical presentations are distinct. ${ }^{2}$

Limitations to this study include small sample size and self-reported disease status (Registry). The rarity of Coats syndrome in an already rare disease makes definitive association studies impracticable.

Coats syndrome occurs most frequently in female patients with FSHD with large contractions (allele size $<15 \mathrm{~kb}$ ) and with variable age at onset (range $<1-53$ years). Recent expert opinion-based FSHD guidelines recommend a single dilated screening eye examination in adults and yearly examinations in infantile-onset disease. ${ }^{1}$ The data presented here suggest that rather than patient age or disease severity, contraction size should determine the need for repeated retinal examinations. Consequently, although we still recommend all patients be screened for retinal vascular involvement at the time of diagnosis, patients with large contractions should be examined yearly afterwards, with a low threshold for fluorescein angiography.

\section{AUTHOR CONTRIBUTIONS}

Jeffrey Statland and Rabi Tawil: drafting/revising the manuscript for content, study concept or design, analysis or interpretation of data, acquisition of data, and statistical analysis. Constantine Farmakidis: drafting/revising the manuscript for content, study concept or design, analysis or interpretation of data. Sabrina Sacconi: drafting/revising the manuscript for content, analysis or interpretation of data, acquisition of data. Mina Chung: drafting/revising the manuscript for content, analysis or interpretation of data, acquisition of data. Colleen DonlinSmith: study concept or design, study coordination.

\section{ACKNOWLEDGMENT}

The authors thank all the people who took the time to complete the survey, in particular Pauline Lahaut, Kathy Mathews, John Kissel, Robin Fitzsimons, and Carsten Bonnemann, the National FSHD Registry, and all the people who gave their time and opinions.

\section{STUDY FUNDING}

No targeted funding reported.

\section{DISCLOSURE}

J. Statland is supported by the MDA Clinical Research Training Grant, and is a consultant for Cytokinetics. S. Sacconi, C. Farmakidis, C. Donlin-Smith, and M. Chung report no disclosures. R. Tawil received research support from the NIH National Institute of Neurological Disorders and Stroke 1P01NS069539-01 (co-PI), NIH R01 AR054366 (coinvestigator), NIH 2U54NS048843-08 (coinvestigator), R01 NS061795-01A2 (co-PI), the Fields Center for FSHD, and Neuromuscular Research, and is a consultant for Cytokinetics. Go to Neurology.org for full disclosures.

Received October 5, 2012. Accepted in final form December 12, 2012. 


\section{REFERENCES}

1. Tawil R, van der Maarel S, Padberg GW, van Engelen BG. 171st ENMC international workshop: standards of care and management of facioscapulohumeral muscular dystrophy. Neuromuscul Disord 2010;20:471-475.

2. Shields JA, Shields CL. Review: coats disease: the 2001 LuEsther T. Mertz lecture. Retina 2002;22:80-91.

3. Fitzsimons RB, Gurwin EB, Bird AC. Retinal vascular abnormalities in facioscapulohumeral muscular dystrophy: a general association with genetic and therapeutic implications. Brain 1987;110:631-648.

4. Padberg GW, Brouwer OF, de Keizer RJ, et al. On the significance of retinal vascular disease and hearing loss in facioscapulohumeral muscular dystrophy. Muscle Nerve 1995;2. S73-S80.

5. Funakoshi M, Goto K, Arahata K. Epilepsy and mental retardation in a subset of early onset 4q35-facioscapulohumeral muscular dystrophy. Neurology 1998;50:1791-1794.
6. Nakagawa M, Matsuzaki T, Higuchi I, et al. Facioscapulohumeral muscular dystrophy: clinical diversity and genetic abnormalities in Japanese patients. Intern Med 1997;36: 333-339.

7. van der Maarel SM, Tawil R, Tapscott SJ. Facioscapulohumeral muscular dystrophy and DUX4: breaking the silence. Trends Mol Med 2011;17:252-258.

8. Bass SJ, Sherman J, Giovinazzo V. Bilateral Coats' response in a female patient leads to diagnosis of facioscapulohumeral muscular dystrophy. Optometry 2011;82: 72-76.

9. Bindoff LA, Mjellem N, Sommerfelt K, et al. Severe fascioscapulohumeral muscular dystrophy presenting with Coats' disease and mental retardation. Neuromuscul Disord 2006; 16:559-563.

10. Ganesh A, Kaliki S, Shields CL. Coats-like retinopathy in an infant with preclinical facioscapulohumeral dystrophy. J AAPOS 2012;16:204-206.

\section{Do You Know What is Happening to Neurology on Capitol Hill?}

Congress is making decisions that affect neurologic research funding and the way neurology is practiced in the United States. Only Capitol Hill Report on AAN.com takes you behind Washington's closed doors and shines a light on how your federal legislators are working for-or against-your interests. Read Capitol Hill Report on AAN.com the second and fourth Wednesday of each month. Stay informed. Your work depends on it.

\section{Mitigate Opioid Misuse in Your Practice}

100 people die from drug overdoses every day in the United States. ${ }^{*}$ Learn how to mitigate opioid misuse in your practice with the AAN's newest NeuroPITM performance improvement module on Chronic Opioid Therapy.

- Helps address both the Performance in Practice (PIP) and Continuing Medical Education (CME) components of Maintenance of Certification (MOC), as mandated by the American Board of Psychiatry and Neurology (ABPN)

- Tackles the timely issue of chronic opioid therapy for non-cancer pain

- Offers measures to address strategies for mitigating opioid misuse

- Features educational resources, links to clinical tools, and patient education materials

Visit www.aan.com/view/neuropi today!

${ }^{*}$ CDC. Vital Signs: Overdoses of Prescription Opioid Pain Relievers-United States, 1999-2008. MMWR 2011;60:1-6 